\title{
The contribution to studies of the effect of $\beta$-glucan on plasma viscosity in a rat sepsis model
}

\author{
Meltem Ercan ${ }^{1}$, Semra Ozdemir $^{1}$
}

\begin{abstract}
Objective: Sepsis is a complex pathophysiological process that involves both alterations in the microcirculation and changes in the biochemical and physiological characteristics of the blood constituents and hyperdynamic syndrome characterized by maldistribution of blood flow. Plasma viscosity is a major determinant of capillary blood flow through the microcirculation. $\beta$-glucan is accepted to be one of the most powerful immune response modifiers. The aim of this study was to investigate the possible protective effect of $\beta$-glucan on the levels of plasma viscosity and malondialdehyde (MDA), after rats was exposed to sepsis.

Methods: Sepsis was induced by cecal ligation and perforation (CLP) in 14 male Wistar albino rat. To evaluate this, rats were divided into four groups as sham operated, $\beta$-glucan treated sham operated, CLP and $\beta$-glucan treated CLP. Sixteen hours after operation, rats were decapitated and MDA, plasma viscosity levels were measured.

Results: Plasma viscosity represented, $1.30 \pm 0.12 \mathrm{mPa}$.s in CLP group and $1.35 \pm 0.09 \mathrm{mPa}$.s in CLP+ $\beta$-glucan group. In the CLP group, MDA levels were found to be higher than in control group. When CLP group compared with $C L P+\beta$-glucan group, no significant statistical differences were observed in the levels of plasma viscosity and MDA.

Conclusion: Our results seem that the given dose of $\beta$-glucan was probably insufficient to prevent sepsis induced plasma viscosity.
\end{abstract}

Keywords: Sepsis, plasma viscosity, $\beta$-glucan, MDA, Rat

\section{Introduction}

Severe sepsis and septic shock are the most common causes of death in intensive care units (ICUs), with associated mortality rates of $30-50 \%$. Sepsis is a heterogenous group of syndromes caused by a systemic inflammatory response and is associated with the development of progressive damage in multiple organs. Disturbances in microvascular flow appear to play a crucial role in the impairment of tissue oxygenation that can contribute to multiple organ failure and death. Rheological changes in patients with severe sepsis and septic shock include slowing of capillary blood flow as a result of decreased perfusion pressure and local arteriolar constriction, viscosity alterations, and disturbances of red (RBC) and white (WBC) blood cell rheology $[1,2,3,4]$. Plasma viscosity is a major determinant of capillary blood flow through the microcirculation. Elevated plasma viscosity leads to an increase in blood viscosity, thereby decreasing blood flow in microcirculation. In this way, "elevated plasma viscosity " may contribute to tissue damage by impairing microcirculatory flow [5].
Microvascular dysfunction is one of the key contributors to organ failure and death in sepsis; however the mechanisms linking sepsis with vascular dysfunction is not understood. Sepsis is associated with heightened oxidative stress [6]. Oxidative stress is described as impairment of balance between prooxidant and antioxidant systems. Under physiological conditions, there is equilibrium between oxidants and antioxidants. These oxidants and antioxidants that are produced by organism in order to avoid and protect from the toxic byproducts that are produced as a result aerobic mechanism [7].

Through the sepsis, overproduction of oxygen free radicals leads to weakening of natural scavenging mechanisms, causing implication in endothelial cell damage and multi organ failure [8]. The oxygen free radicals, which include malondialdehyde (MDA), hydrogen peroxide, and hydroxyl radicals, cause "oxidative stress.

Glucans are natural produced as a result of biological response modifiers that are composed of (1-3)- $\beta$-D-linked polymers of glucose. In animal studies glucans have been shown to be effective in

Received 05-01-2015 Accepted 05-01-2015 Avalable Online 10-01-2015

${ }^{1}$ Istanbul University, Cerrahpasa Medical Faculty, Department of Biophysics, Istanbul, Turkey

*Corresponding Author: Semra Ozdemir E-mail: hsf@istanbul.edu.tr 
preventing experimental peritonitis. Clinical studies suggest that intake of glucans in trauma surgical patients will increase the chance of survival via decreasing the frequencies of septic complications that will stimulate conversion of energy.

The mechanism(s) by which glucans alter the septic state is unknown [9]. Several mechanisms were proposed for the protective effect of $\beta$-glucan, one of them is related to antioxidant capacity of the molecule [10]. There are several studies in which the relationships between sepsis and rheological changes have been investigated [4, 11, 12]. However, the $\beta$-glucan's effect over plasma viscosity or its alterations have not been studied.

The aim of this study was to understand the effect of $\beta$-glucan application over the possible changes in plasma viscosity, in sepsis model created on rats by CLP method

\section{Material and Methods}

All experimental protocols were performed in accordance with the National Institutes of Health guidelines and the approval of the Istanbul University, Cerrahpasa Medical Faculty Animal Care and Use Ethics Committee.

\section{Animals and experimental design}

Male Wistar albino rats, weighing 200-250 $\mathrm{g}$ were kept in individual wire-bottom cages, in a room at a constant temperature $\left(22 \pm 2^{\circ} \mathrm{C}\right)$ with 12 -h light and dark cycles, and fed standard rat chow. The rats were divided into the following four groups of seven rats each: sham operated control (C), sham operated $+\beta$-glucan treated group (Glucan), cecal ligation and perforation (CLP) group, and cecal ligation and perforation $+\beta$-glucan treated $(\beta$-glucan+CLP) group. Sepsis was induced by CLP (cecal ligation and perforation) technique [13,14 ]. (Figure 1). General anesthesia was induced by injection of intraperitoneal ketamine hydrochloride (Ketalar, Parke-Davis, USA). All procedures were performed under sterile conditions. The antimesentric surface of the cecum was perforated with an 22 gauge needle at two locations $1 \mathrm{~cm}$ apart and the cecum was gently compressed until fecal matter was extruded and then returned to the abdominal cavity and the laparotomy was closed with 4.0 silk sutures. The sham procedure consisted of identification, exteriorization, and then replacement of the cecum within the peritoneal cavity. The incision in both cases was closed in two layers. At the end of the surgical procedure, the rats were allowed to recover from anesthesia with free access to rat chow and water. After operation, all rats were resuscitated with saline, $3 \mathrm{ml} / 100 \mathrm{~g}$ body weight given subcutaneously. Postoperatively, the rats were then returned to their cages with free access to food and water. After the CLP or sham procedure was performed $(2 \mathrm{~h})$, animals were reviewed for clinical signs of the development of sepsis. All CLP animals were lethargic; showed lack of interest in their environment, food, and hygiene; displayed piloerection; and had crusty exudates around their eyes. In contrast, the sham animals were healthy, moving freely, and eating.

\section{$\beta$-glucan treatment}

The $\beta$-glucan (Mustafa Nevzat Company, Turkey) we used in this study is $1,3-1,6 \beta-\mathrm{D}$ glucan in the microparticulate form which is prepared from Saccharomyces cerevisiae yeast. $\beta$ glucan was suspended in saline. $\beta$-glucan $50 \mathrm{mg} / \mathrm{kg}$ per oral (po) was given by intragastric gavage once a day for 10 days and $30 \mathrm{~min}$ before and also $6 \mathrm{~h}$ after the operation [14,15]. The rats were decapitated $16 \mathrm{~h}$ after the CLP procedure, bloods were taken and samples were stored at $-70^{\circ} \mathrm{C}$.

\section{Measurement of thiobarbituric acid reactive species (TBARS)}

Thiobarbituric acid (TBA) test was applied for MDA level measurement that is the final product of lipid peroxidation. Measurements were done according to Uchiyama and Mihara method [16]. Absorbance's were read at $532 \mathrm{~nm}$. 1, 1, 3, 3Tetra-ethoxypropane was used as the standard. Results were calculated as $\mathrm{nmol} \mathrm{MDA} / \mathrm{ml}$ in plasma.

\section{Measurement of plasma viscosity (PV)}

Blood samples for plasma viscosity measurements were drawn into vacutainers with potassium EDTA as anticoagulant and were posed in two hours following collection in accordance with the committee of hemorheology standardization (1986). The viscosity of a liquid depends on its temperature, and for most fluids viscosity decreases as temperature increases. The viscosity of a liquid can be measured by a viscometer, which is a device built for studying stress strain relations. Capillary viscometers are the most widely used devices for measuring viscosity of Newtonian liquids. The working principle of a capillary viscometer is based on the measurement of flow rate of the liquid through a well-defined capillary tube under a certain pressure difference; at constant temperature and pressure difference; the flow rate decreases as viscosity increases. Plasma viscosity was measured from plasma isolated from each subject after the subject's blood was centrifuged for $10 \mathrm{~min}$ at $3.000 \mathrm{rpm}$ and the plasma and buffy coat were separated. Plasma viscosity was measured by Harkness capillary viscometer (Coulter Electronics LTD Serial Number 6083, England) at $37^{\circ} \mathrm{C}$ [17]. For the measurement of blood plasma, a specialized Harkness capillary viscometer has been recommended by Baskurt O.K. [18] which allows measurement of sizes as low as $0.5 \mathrm{ml}$ within $1 \mathrm{~min}$. The flow rate, measured in seconds (s), of each plasma sample (Tp) was compared with that of distilled water (Tw) to obtain 
the relative plasma viscosity (coefficient of variation, $1.00 \%$ ). For quality control measurements were compared with daily water. Plasma viscosity measurements were carried out in triplicate. The plasma viscosity was expressed as in milliPascal $\times$ seconds $(\mathrm{mPa} . \mathrm{s})$ which is numerically equal to centipoise $(\mathrm{cP})$;

$$
P_{v}=\eta_{w} \frac{T_{p}(s)}{T_{w}(s)}
$$

$\eta_{\mathrm{w}}=0.693 \mathrm{mPa} . \mathrm{s}$ (Constant value for water viscosity at $37^{\circ} \mathrm{C}$ )

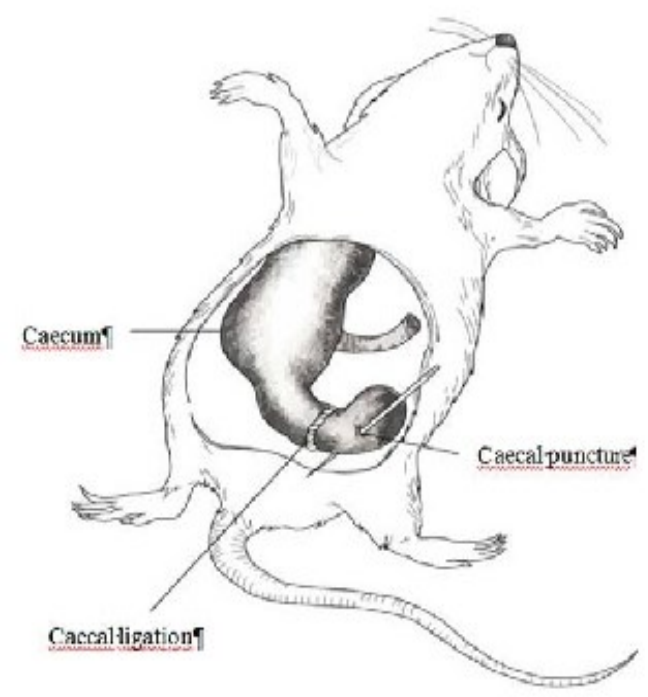

Figure 1. CLP (cecal ligation and perforation) technique

\section{Statistical Analysis}

All data are expressed as mean \pm standard deviation (SD). Statistical analyses were performed with Statistical Products and Service Solution package (SPSS, for Windows, 10.0.1 version, Chicago, III., USA). Significance of the differences was tested by analysis of variance (one-way ANOVA), followed by Tukey's test to determine statistical differences between groups. Differences were considered statistically significant when the $p$ value was $<0.05$

\section{Results}

The levels of plasma viscosity represented, $1.58 \pm 0.17 \mathrm{mPa} . \mathrm{s}$ in control group, $1.53 \pm 0.15$ $\mathrm{mPa} . \mathrm{s}$ in $\beta$-glucan group, $1.30 \pm 0.12 \mathrm{mPa} . \mathrm{s}$ in CLP group and $1.35 \pm 0.09 \mathrm{mPa} . \mathrm{s}$ in $\mathrm{CLP}+\beta$-glucan group. In the CLP group, the levels of plasma viscosity were found to be significantly lower than in control group $(p<0.001)$. In the $\mathrm{CLP}+\beta$-glucan group, plasma viscosity $(p<0.01)$, were found to be significantly lower than those of controls. When
CLP group compared with CLP $+\beta$-glucan group, no significant statistical differences were observed in the levels of the plasma viscosity $(p>0.05)$, (Table $1)$. In the CLP group, plasma MDA levels were found to be significantly higher than in control group $(p<0.001)$. In the $C L P+\beta$-glucan group, MDA levels of plasma $(p<0.001)$, were found to be significantly higher than those of controls. When CLP group compared with CLP $+\beta$-glucan group, no significant statistical differences were observed in MDA levels of the plasma $(p>0.05)$ (Table 1).

\section{Discussion}

Sepsis is associated with abnormalities in systemic and microcirculatory blood flow, decreased red blood cell (RBC) deformability and impaired oxygen delivery leading to tissue hypoperfusion and tissue dysfunction with subsequent cardiovascular changes [19]. Sepsis is characterized by a number of circulatory disorders, including decreased systemic vascular resistance, hypotension, impaired oxygen utilization, lactic acidosis, and maldistribution of blood flow in the microcirculation [6]. Alterations in microvascular blood flow and oxygenation have been demonstrated in various models of sepsis. In a normodynamic septic model using cecal ligation and puncture (CLP) in rats, reduced perfused capillary density and increased heterogeneity have been observed in striated muscles and intestinal mucosa [20].

The impairment leads to tissue hypoxia due to increased diffusion distance for oxygen. It has been proposed that hypoxia is the initial and important step towards organ failure. Maldistribution of blood flow within the capillary bed is one of the hallmarks of sepsis. This dysfunction is associated with oxygen transport impairment within tissues and may precipitate organ failure [21]. Because the microcirculation is essentially adaptive, it is important to understand whether the sepsis-associated alterations are the primary event leading to cellular dysfunction or the changes in perfusion reflect directly altered cellular metabolism (adaptive theory). In addition, oxygen saturation at the capillary end of well-perfused capillaries is low, suggesting that the tissues are using the delivered oxygen [22].

Distribution of blood flow through the many parallel, small vessels of the microcirculation is of vital importance for organ function. For a given vascular architecture, flow resistance is determined by the rheological behavior of the blood flowing through microvessels. The mechanisms by which this blood flow is regulated include myogenic factors (i.e., strain and stress forces, via blood and plasma viscosity), metabolic factors such as $\mathrm{pH}$, $\mathrm{pCO}_{2}$ and potassium, $\mathrm{O}_{2}$, and nitric oxide (NO) and neurohormonal control [23]. 
Table 1. The levels of plasma MDA and viscosity in control, $\beta$-glucan, cecal ligation and perforation (CLP) and $C L P+\beta$-glucan groups

PV: plasma viscosity, MDA: malondialdehyde
Results were calculated as nmol MDA $/ \mathrm{ml}$ in plasm

\begin{tabular}{ccccc}
\hline & $\begin{array}{c}\text { Control } \\
(\mathrm{n}=7)\end{array}$ & $\begin{array}{c}\beta \text {-Glucan } \\
(\mathrm{n}=7)\end{array}$ & $\begin{array}{c}\text { CLP } \\
(\mathrm{n}=7)\end{array}$ & $\begin{array}{c}\text { CLP+ } \beta \text {-Glucan } \\
(\mathrm{n}=7)\end{array}$ \\
\hline MDA(nmol $/ \mathrm{ml})$ & $3.45 \pm 0.31$ & $3.27 \pm 0.34$ & $7.30 \pm 0.54 * * *$ & $7.26 \pm 0.30 * * *$ \\
$\mathrm{PV}(\mathrm{mPa} . \mathrm{s})$ & $1.58 \pm 0.17$ & $1.53 \pm 0.15$ & $1.30 \pm 0.12 * * *$ & $1.35 \pm 0.09 * *$ \\
\hline
\end{tabular}

Values are expressed as Means $\pm \mathrm{SD}$. ** $p<0.01, * * * p<0.001$ versus control group

Hemorheology is the study of deformation and flow of blood and blood cells. The prime function of blood is transport by flow, and the most important rheological property of blood is its resistance to flow, or viscosity. Since the viscosity of blood is a major determinant of normal blood flow throughout the circulation, any increase in viscosity will cause an impairment of the normal flow. Whole blood viscosity is affected by four parameters: hematocrit levels, erythrocyte aggregability, erythrocyte deformability and plasma viscosity[ 4].

Plasma is the suspending phase for the cellular elements in blood, and thus a change in its viscosity directly affects blood viscosity regardless of the hematocrit and the properties of the cellular elements. Plasma is a Newtonian fluid (i.e., viscosity independent of shear rate), yet technical artifacts have led some to report non-Newtonian behavior of plasma. If the value of plasma viscosity is normal, it is not a specific parameter that reflects the disease process. In physiological situations that are related to acute phase reactions and/or, value of plasma viscosity may differ due to a change of proteins in size and in number. Under these conditions plasma viscosity can be used as a parameter. Plasma proteins influence both whole blood viscosity and red cell aggregation. Plasma proteins exert their effects either directly or through their influence on red cell aggregation [24].

Changes in fibrinogen and immunoglobulins, under both physiologic and pathologic conditions can increase whole blood viscosity. Blood flow through the microvasculature is impaired when viscosity increases, leading to tissue ischemia. Abnormalities of fibrinogen greatly increase its ability to cause red cell aggregation, and are a contributory pathogenic factor in ischemic heart disease and stroke. Immunoglobulins may affect blood viscosity directly, or by increasing the red cell aggregation $[4,25]$.

Sepsis induces profound changes in the microcirculation with loss of capillary density, maldistribution of blood flow, increased flow heterogeneity, changes in microvascular reactivity, and $\mathrm{WBC}$-endothelial cell adhesion and vascular leakage. $[1,11,22,26]$.

In our previous study in which showed that sepsis may occur with histopathological methods, we benefited from CLP method for creating a sepsis. Thereby it is shown that it is possibility of creating sepsis with CLP method was shown in histopathologically [27]. In this study, the values of plasma viscosity represented $1.58 \pm 0.17 \mathrm{mPa} . \mathrm{s}$ in the control group. These results are consistent with Windberger $U$. et al. [28]. A statistically significance was seen in values of the plasma viscosity of control group and CLP group. As far as we could reach, there has been no study measuring plasma viscosity in experimental sepsis by inducing CLP technique.

During the course of severe sepsis, several biochemical abnormalities develop, causing a change in metabolic pathways of various organs that result in secondary damage. MDA is the breakdown product of the major chain reactions leading to oxidation of polyunsaturated fatty acids, and thus serves as a reliable marker of oxidative stress-mediated lipid peroxidation. An increased concentration of MDA reflects the level of lipid peroxidation in tissues and plasma [29].

Sepsis is associated with heightened oxidative stress. There is evidence that oxidative stress has an important role in the development of sepsis-induced multiorgan failure. Diminished antioxidative defences, superoxide dismutase, catalase and glutathione, also contribute to oxidative stress. Recent studies have reported increased levels of lipid peroxides and decreased antioxidant enzyme activity in experimental sepsis, indicating an exhaustion of the antioxidant system [30, 31, 32]. Bateman et al. [32] reported that there was no significant difference in TBARS levels of normal and CLP exposed animals.

Ritter et al. [33] showed that MDA and plasma superoxide dismutase levels are markers of early mortality in septic rats. Batra et al. [ 34] found that MDA levels were also increased in neonates with sepsis suggesting that the elevations of these antioxidant enzymes were not so effective as to prevent cellular damage Enhancement of antioxidant status in animal models of sepsis has demonstrated beneficial effect and administration of antioxidants to septic patients may be protective.

The beneficial effects on the immune system and the lack of toxic or adverse effect had focused the studies on $\beta$-glucan molecule. Some researchers have claimed that protective effect was due to the antioxidant capacity. Furthermore, it has been suggested that $\beta$-glucan binds to scavenger receptors Brown and Gordon and Lazarová et al. $[35,36]$ observed protective effects of 
carboxymethyl chitin-glucan (dose approximately $200 \mathrm{mg} / \mathrm{kg}$ body weight for 21 days) supplementation in term of the decreased level of DNA damage measured by comet assay in different primary rat cells damaged after isolation either with genotoxic carcinogens requiring metabolic activation or oxidative stress induced by visible light-excited methylene blue. Lazarová et al. [37] also, showed immunopotentiating activity of $\beta$-Dglucans, efficiently inhibiting the genotoxicity of carcinogens requiring metabolic activation. Sener et al. [10] showed that treatment with $\beta$-glucan significantly reversed the elevations in MDA levels in the liver, kidney, heart, lung, diaphragm and brain tissues. However, the relationship between the plasma viscosity and plasma MDA levels effects of $\beta$-glucan administration in sepsis has not been defined.

We examined the effects of $\beta$-glucan therapy on the plasma viscosity and MDA levels in experimental sepsis. The results of the present study demonstrate that sepsis causes a decrease in the levels of plasma viscosity and an increase in the levels of plasma MDA. In our previous study, we observed that kidney and liver tissues of control and $\beta$-glucan groups show similarity in histological changes. $\beta$-glucan treatment was not effective in correcting histological changes seen in the subjects of the sepsis group [27].

Our results indicated that $\beta$-glucan administration did not return the levels of plasma viscosity and MDA to the control group level. It seems likely that the given dose of $\beta$-glucan was insufficient to prevent sepsis induced the level of plasma viscosity and MDA. In order to determine the effect of $\beta$-glucan, the study should be carried out with different doses and lengths.

Financial Support: This research received no specific grant from any funding agency, commercial or not-for-profit sectors

Conflict of Interest: The authors declare no potential conflicts of interest with respect to the research, authorship, and/or publication of this article.

\section{References}

1. Piagnerelli M, Boudjeltia KZ, Vanhaeverbeek M, Vincent JL. Red blood cell rheology in sepsis. Intensive care medicine. 2003;29(7):1052-61.

2. Aird WC. The role of the endothelium in severe sepsis and multiple organ dysfunction syndrome. Blood. 2003;101(10):3765-77.

3. Kirschenbaum LA, Aziz M, Astiz ME, Saha DC, Rackow EC. Influence of rheologic changes and platelet-neutrophil interactions on cell filtration in sepsis. American journal of respiratory and critical care medicine. 2000;161(5):1602-7.
4. Baskurt OK, Meiselman HJ. Blood rheology and hemodynamics. Seminars in thrombosis and hemostasis. 2003;29(5):435-50.

5. Napoli C, de Nigris F, Williams-Ignarro S, Pignalosa O, Sica V, Ignarro LJ. Nitric oxide and atherosclerosis: an update. Nitric oxide : biology and chemistry / official journal of the Nitric Oxide Society. 2006;15(4):265-79.

6. Tyml K. Critical role for oxidative stress, platelets, and coagulation in capillary blood flow impairment in sepsis. Microcirculation. 2011;18(2):152-62.

7. Tsai K, Hsu T, Kong C, Lin K, Lu F. Is the endogenous peroxyl-radical scavenging capacity of plasma protective in systemic inflammatory disorders in humans? Free radical biology \& medicine. 2000;28(6):926-33.

8. Peralta JG, Llesuy S, Evelson P, Carreras MC, Flecha BG, Poderoso JJ. Oxidative stress in skeletal muscle during sepsis in rats. Circulatory shock. 1993;39(2):153-9.

9. Williams DL, Ha T, Li C, Kalbfleisch JH, Laffan JJ, Ferguson DA. Inhibiting early activation of tissue nuclear factor-kappa B and nuclear factor interleukin 6 with (1-->3)-beta-D-glucan increases long-term survival in polymicrobial sepsis. Surgery. 1999;126(1):54-65.

10. Sener G, Toklu H, Ercan F, Erkanli G. Protective effect of beta-glucan against oxidative organ injury in a rat model of sepsis. International immunopharmacology. 2005;5(9):1387-96.

11. Ellis CG, Bateman RM, Sharpe MD, Sibbald WJ, Gill R. Effect of a maldistribution of microvascular blood flow on capillary $\mathrm{O}(2)$ extraction in sepsis. American journal of physiology Heart and circulatory physiology. 2002;282(1):H156-64

12. Baskurt OK, Gelmont D, Meiselman HJ. Red blood cell deformability in sepsis. American journal of respiratory and critical care medicine. 1998;157(2):421-7.

13. Wichterman KA, Baue AE, Chaudry IH. Sepsis and septic shock--a review of laboratory models and a proposal. The Journal of surgical research. 1980;29(2):189-201.

14. Ozdemir S, Toplan S, Ercan M, Bayrak I, Uruluer B, Sunamak O. The effect of beta-glucan on trace element levels in intra-abdominal sepsis in rats. Biological trace element research. 2009;132(1-3):197-206.

15. Senoglu N, Yuzbasioglu MF, Aral M, Ezberci M, Kurutas EB, Bulbuloglu E, et al. Protective effects of $\mathrm{N}$-acetylcysteine and beta-glucan pretreatment on oxidative stress in cecal ligation and puncture model of sepsis. Journal of investigative surgery : the official journal of the Academy of Surgical Research. 2008;21(5):237-43.

16. Mihara M, Uchiyama M. Determination of malonaldehyde precursor in tissues by thiobarbituric acid test. Analytical biochemistry. 1978;86(1):271-8.

17. Harkness J. A new instrument for the measurement of plasma-viscosity. Lancet. 1963;2(7302):280-1.

18. Baskurt OK, Boynard M, Cokelet GC, Connes P, Cooke $\mathrm{BM}$, Forconi $\mathrm{S}$, et al. New guidelines for hemorheological laboratory techniques. Clinical hemorheology and microcirculation. 2009;42(2):75-97. 
19. Moutzouri AG, Skoutelis AT, Gogos CA, Missirlis YF, Athanassiou GM. Red blood cell deformability in patients with sepsis: a marker for prognosis and monitoring of severity. Clinical hemorheology and microcirculation. 2007;36(4):291-9.

20. Matsuda N, Hattori Y. Vascular biology in sepsis: pathophysiological and therapeutic significance of vascular dysfunction. Journal of smooth muscle research $=$ Nihon Heikatsukin Gakkai kikanshi. 2007;43(4):117-37.

21. Lam C, Tyml K, Martin C, Sibbald W. Microvascular perfusion is impaired in a rat model of normotensive sepsis. The Journal of clinical investigation. 1994;94(5):2077-83.

22. De Backer D, Donadello K, Taccone FS, OspinaTascon G, Salgado D, Vincent JL. Microcirculatory alterations: potential mechanisms and implications for therapy. Annals of intensive care. 2011;1(1):27.

23. Ince C. The microcirculation is the motor of sepsis. Critical care. 2005;9 Suppl 4:S13-9.

24. Kesmarky G, Kenyeres P, Rabai M, Toth K. Plasma viscosity: a forgotten variable. Clinical hemorheology and microcirculation. 2008;39(1-4):243-6.

25. Pries AR, Secomb TW. Rheology of the microcirculation. Clinical hemorheology and microcirculation. 2003;29(3-4):143-8.

26. Hinshaw LB. Sepsis/septic shock: participation of the microcirculation: an abbreviated review. Critical care medicine. 1996;24(6):1072-8.

27. Ozdemir S, Toplan S, Tanriverdi G, Sunamak O. The effects of beta-glucan on iron levels and lipid peroxidation in intra-abdominal sepsis in rats. General physiology and biophysics. 2011;30(2):138-44.

28. Windberger U, Bartholovitsch A, Plasenzotti R, Korak KJ, Heinze G. Whole blood viscosity, plasma viscosity and erythrocyte aggregation in nine mammalian species: reference values and comparison of data. Experimental physiology. 2003;88(3):431-40.
29. Tekin A, Kucukkartallar T, Turkyilmaz S, Dinckan A, Esen H, Ates B, et al. Effects of caffeic acid phenethyl ester (CAPE) on sepsis in rats. Inflammation. 2008;31(4):273-80.

30. Andrades M, Ritter C, Moreira JC, Dal-Pizzol F. Oxidative parameters differences during non-lethal and lethal sepsis development. The Journal of surgical research. 2005;125(1):68-72

31. Liaw WJ, Chen TH, Lai ZZ, Chen SJ, Chen A, Tzao C, et al. Effects of a membrane-permeable radical scavenger, Tempol, on intraperitoneal sepsis-induced organ injury in rats. Shock. 2005;23(1):88-96.

32. Bateman RM, Jagger JE, Sharpe MD, Ellsworth ML, Mehta S, Ellis CG. Erythrocyte deformability is a nitric oxide-mediated factor in decreased capillary density during sepsis. American journal of physiology Heart and circulatory physiology. 2001;280(6):H2848-56.

33. Ritter C, Andrades M, Frota Junior ML, Bonatto F, Pinho RA, Polydoro M, et al. Oxidative parameters and mortality in sepsis induced by cecal ligation and perforation. Intensive care medicine. 2003;29(10):17829.

34. Batra S, Kumar R, Kapoor AK, Ray G. Alterations in antioxidant status during neonatal sepsis. Annals of tropical paediatrics. 2000;20(1):27-33.

35. Brown GD, Gordon S. Fungal beta-glucans and mammalian immunity. Immunity. 2003;19(3):311-5.

36. Lazarova M, Labaj J, Kogan G, Slamenova D. Carboxymethyl chitin-glucan enriched diet exhibits protective effects against oxidative DNA damage induced in freshly isolated rat cells. Neoplasma. 2006;53(5):434-9.

37. Lazarova M, Labaj J, Eckl P, Kogan G, Slamenova D. Effects of dietary intake of a fungal beta-D-glucan derivative on the level of DNA damage induced in primary rat hepatocytes by various carcinogens. Nutrition and cancer. 2006;56(1):113-22. 